\title{
Laser-only Adaptive Optics Achieves Significant Image Quality Gains Compared to Seeing-limited Observations over the Entire Sky
}

\author{
Ward S. Howard ${ }^{1}$ (D), Nicholas M. Law ${ }^{1}$ (D), Carl A. Ziegler ${ }^{1}$ (D), Christoph Baranec ${ }^{2}$ (D), and Reed Riddle ${ }^{3}$ (iD) \\ ${ }^{1}$ Department of Physics and Astronomy, University of North Carolina at Chapel Hill, Chapel Hill, NC 27599-3255, USA; wshoward@unc.edu \\ ${ }^{2}$ Institute for Astronomy, University of Hawai'i at Mānoa, Hilo, HI 96720-2700, USA \\ ${ }^{3}$ Division of Physics, Mathematics, and Astronomy, California Institute of Technology, Pasadena, CA 91125, USA \\ Received 2017 August 9; revised 2017 November 8; accepted 2017 November 12; published 2018 January 15
}

\begin{abstract}
Adaptive optics laser guide-star systems perform atmospheric correction of stellar wavefronts in two parts: stellar tip-tilt and high-spatial-order laser correction. The requirement of a sufficiently bright guide star in the field-ofview to correct tip-tilt limits sky coverage. In this paper, we show an improvement to effective seeing without the need for nearby bright stars, enabling full sky coverage by performing only laser-assisted wavefront correction. We used Robo-AO, the first robotic AO system, to comprehensively demonstrate this laser-only correction. We analyze observations from four years of efficient robotic operation covering 15000 targets and 42000 observations, each realizing different seeing conditions. Using an autoguider (or a post-processing software equivalent) and the laser to improve effective seeing independent of the brightness of a target, Robo-AO observations show a $39 \% \pm 19 \%$ improvement to effective FWHM, without any tip-tilt correction. We also demonstrate that 50\% encircled energy performance without tip-tilt correction remains comparable to diffraction-limited, standard Robo-AO performance. Faint-target science programs primarily limited by $50 \%$ encircled energy (e.g., those employing integral field spectrographs placed behind the AO system) may see significant benefits to sky coverage from employing laser-only AO.
\end{abstract}

Key words: atmospheric effects - instrumentation: adaptive optics - instrumentation: high angular resolution techniques: high angular resolution - techniques: image processing

\section{Introduction}

Correcting atmospheric distortion of stellar wavefronts involves two components: tip-tilt (e.g., stellar image displacement) and point-spread function (PSF) irregularities. The image quality of laser adaptive optics suffers without a sufficiently bright guide star nearby to correct tip-tilt error (Rigaut \& Gendron 1992). High-resolution-imaging science programs that include faint targets are thus susceptible to tip-tilt errors.

In the case of Kepler Objects of Interest (KOIs, i.e., stars that host planet candidates), follow-up using high-resolution observations is a key step to rule out false-positive scenarios. Kepler looks for the dip in brightness due to transits of the planet in front of the host star, and uses the transit depth and stellar radius to estimate planetary radius (O'Donovan et al. 2006; Koch et al. 2010; Law et al. 2014). Any blending of associated stars on the sky to the host dilute the transit depth and artificially decrease the calculated planetary radius (Johnson et al. 2011). Ziegler et al. (2017) notes that faint hosts have received less focus in high-resolution follow-up efforts.

For example, M-dwarfs, the most populous stellar type in the Galaxy (Chabrier 2003), are a class of intrinsically faint stars; Earth-sized planets orbit within the habitable zones of approximately one in six M-dwarfs (Dressing \& Charbonneau 2015). Although M-dwarfs account for only $2 \%$ of all KOIs, the percentage of M-dwarfs among the faintest $12 \%$ of KOIs is three times higher than in the overall KOI population. ${ }^{4}$ The ability to detect companion stars among the faintest KOIs disproportionately affects the confirmation of M-dwarf rocky planets.

\footnotetext{
4 https://exoplanetarchive.ipac.caltech.edu/
}

A KOI companion-star survey with Robo-AO, the first autonomous laser guide-star (LGS) adaptive optics system (Baranec et al. 2014), has already observed 3857 KOIs due to its low observation overheads (Law et al. 2014; Baranec et al. 2016b; Ziegler et al. 2017). With hundreds of KOIs too faint for full Robo-AO post-facto image registration $\left(m_{V}>15.5\right)$, even modest gains in resolution above the seeing-limit allow discovery of companions deep within the Kepler $\sim 4^{\prime \prime}$ pixel scale (Haas et al. 2010).

In order to increase AO coverage of faint targets, methods to minimize tip-tilt error have been developed. The standard approaches are natural guide-star (NGS) AO, LGS AO (Foy \& Labeyrie 1985), off-axis tip-tilt correction, e.g., (Steinbring et al. 2005; Trujillo et al. 2013; Wizinowich et al. 2014), and laser-only AO (Davies et al. 2008). NGS AO employs bright guide stars to correct both tip-tilt and high-order errors. LGS AO supplies a LGS to correct high-order errors, although an NGS is still necessary to correct tip-tilt. Off-axis tip-tilt correction employs an off-axis guide star with respect to the observation target for approximate tip-tilt correction. Laseronly AO employs an LGS AO system, but with no natural guide star, and hence no tip-tilt correction.

Correcting high-order errors requires a brighter guide star than does correcting tip-tilt, vastly expanding the sky coverage of LGS AO over NGS AO. Even with LGS, the necessity of sufficiently bright guide stars significantly limits AO sky coverage (Rigaut \& Gendron 1992).

For large apertures, off-axis tip-tilt error is reduced compared to smaller 1 or $2 \mathrm{~m}$ aperture AO systems (Hardy 1998). Offaxis guide stars approximate the tip-tilt of the target star, improving effective seeing. Using the Altair AO system on the Gemini North telescope (Roberts \& Singh 1998), Trujillo et al. (2013) designed and tested LGS + Peripheral WFS 1 (LGS 

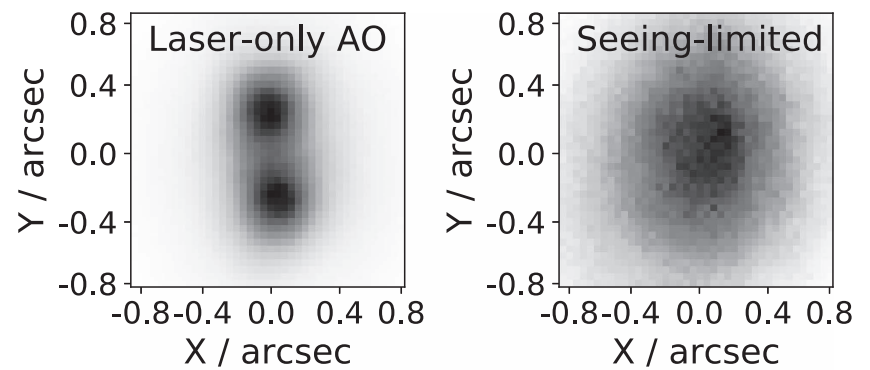

(a) binary star
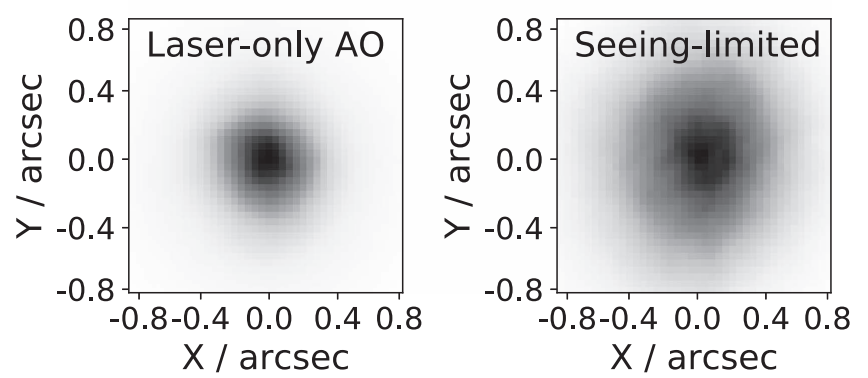

(b) single star

Figure 1. No tip-tilt + laser observations from GenSTAC, typifying improvement above the seeing-limit for a binary star 1(a) and a single star 1(b). Both images are selected from the one-second (no tip-tilt correction), FWHM median-improvement bin of Figure 6(c) and displayed with min-max scaling.

$+\mathrm{P} 1$ henceforth), an off-axis guiding mode with low-Strehl "super-seeing." LGS + P1 NIR observations of $10^{1}-10^{2}$ targets give 2-3× improvement in effective seeing (Trujillo et al. 2013). However, off-axis guiding is intended for large apertures, as scintillation and tip-tilt anisoplanatism decrease as aperture increases.

Also relying upon reduced tip-tilt for large apertures, Davies et al. (2008) and references therein explore the use of an LGS system while foregoing the tip-tilt guide star entirely. Employing the VLT in K-band for a handful of observations, they demonstrate significant improvement to FWHM and encircled energy using laser-only AO.

Off-axis guiding is less applicable to AO systems on intermediate-class telescopes, such as on the Palomar 60 inch (Baranec et al. 2014) or Kitt Peak $2.1 \mathrm{~m}$ (Jensen-Clem et al. 2018), the respective past and present host telescopes to Robo-AO. Scintillation also impacts the performance of laseronly AO on smaller apertures more than on larger telescopes, but the loss in effective seeing has been poorly characterized for large numbers of targets on smaller telescopes.

We evaluate the effectiveness of laser-only AO systems without tip-tilt correction as an approach to sky coverage limitations for faint targets, but do so for $10^{4}$ targets, using a vastly smaller robotic LGS system than the VLT or Gemini North and in the visible instead of NIR. To do this, we employ a new observation pipeline, Generalized Stellar Tracking And Correction (GenSTAC), described in Section 2.2 of this paper. Robo-AO+GenSTAC (see Figure 1) reaches targets from the Robo-AO guide-star limiting magnitude of $\sim m_{V}=15.5$ to the telescope limiting magnitude for a given exposure time. GenSTAC achieves sky coverage at visible wavelengths through its ability to point anywhere in the sky, but at the cost of reduced angular resolution. For arbitrarily long exposures, GenSTAC would need to be replaced with a physical autoguider to best perform laser-only correction.
GenSTAC converts stars that are too faint for guide-star correction into sufficiently bright stars through stacking of an integer number of $N$ binned frames and cubic-spline interpolation of stellar drift between averaged stellar positions. Any tiptilt information inherent in brighter targets is applied for increased improvement to seeing, up to the diffraction limit, much as the current Robo-AO faint-star (i.e., $15.5<m_{V}<18$ ) pipeline at Kitt Peak does (Jensen-Clem et al. 2018). When GenSTAC is instead applied to truly photon-starved targets with no remaining tip-tilt information, laser-only AO provides constant improvement down to the telescope limiting magnitude for a given exposure time.

Although GenSTAC's primary purpose is to simulate an autoguider in order to test laser-only AO, it becomes a tip-tilt optimizer when the number of averaged frames is close to one. In this limit, other image registration methods designed for the background-dominated regime of at least a few photons per frame, e.g., (Snyder \& Schulz 1990; Guillaume et al. 1998; Gratadour et al. 2005), may outperform GenSTAC when applied to targets bright enough for partial tip-tilt correction.

In Section 2, we describe our observational setup and GenSTAC pipeline design. We also describe our empirical measurements of laser correction with the Robo-AO database. In Section 3, we describe the results of our measurement of the relative contributions of tip-tilt and laser correction; we also describe the performance of GenSTAC from targets bright enough for full tip-tilt correction to laser-only targets at the limiting magnitude of the telescope for a given exposure time. In Section 4, we conclude and provide recommendations for the future use of laser-only AO.

\section{Methods}

To measure the performance of operating in a laser-only mode, we first reduce observations from the Robo-AO system with our laser-only pipeline.

\subsection{Observations and Instrument Setup}

Observations were acquired from 2012 May through 2015 June with Robo-AO, mounted on the Palomar 60 inch $(1.5 \mathrm{~m})$ telescope. Robo-AO efficiently observes hundreds of targets in a night (Baranec et al. 2014). As such, the observed-targets database resulting from hundreds of nights over four years of Robo-AO operation allows quantification of $\mathrm{AO}$ performance on a large scale.

While the AO system sets up for a given target, it obtains a $20 \mathrm{~s}$ seeing-limited observation to assess seeing conditions. The laser-launch system then pulses a $12 \mathrm{~W}, 355 \mathrm{~nm}$ ultraviolet beam along the host telescope line-of-sight out to a distance of $10 \mathrm{~km}$ to obtain a measurement of the atmospheric turbulence wavefront. A Shack-Hartmann wavefront sensor in the adaptive optics $1.2 \mathrm{kHz}$ control loop feeds information to a CPU driving a MEMS actuator system, which adapts the shape of a deformable mirror in the optical path, providing wavefront correction. An Andor iXon EMCCD science camera records images at 8.6 FPS (0.1168 s frametime), allowing sufficiently short frametimes for after-the-fact guide-star correction of tiptilt errors, in a reduction pipeline described in Law et al. (2014). The EMCCD reduces the read noise for short frametimes from about $50 e^{-}$to $<1 e^{-}$; Robo-AO employs typical EM-gains between $25 \times$ and $300 \times$. The raw data are 


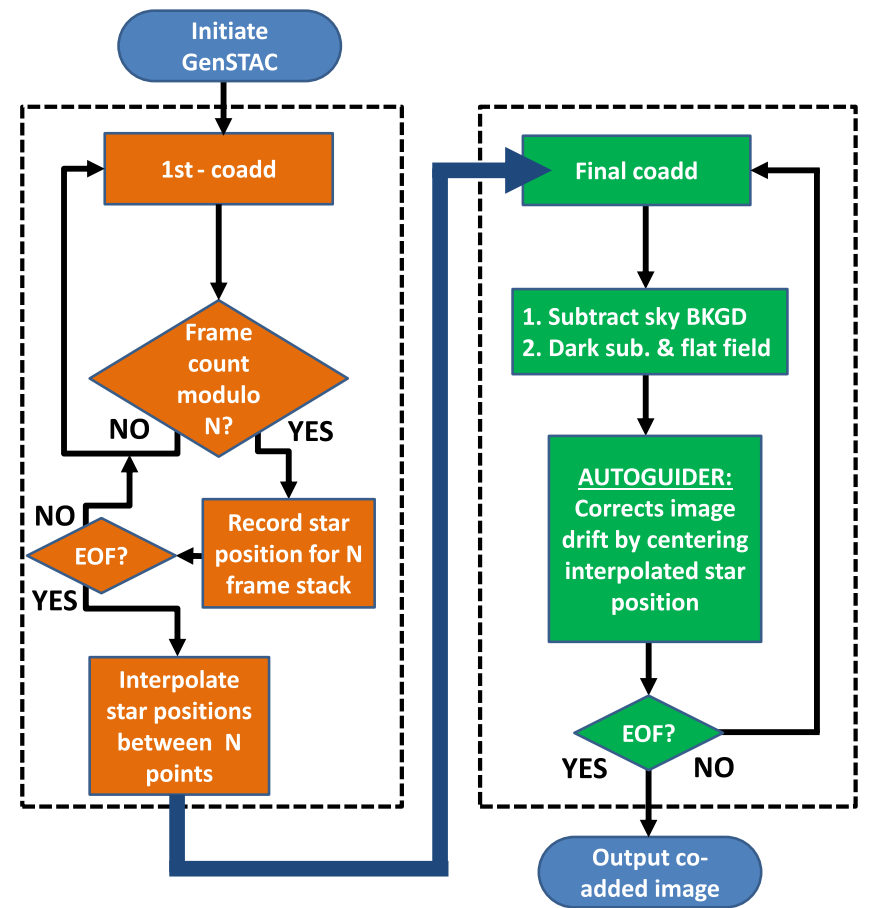

Figure 2. A flowchart describing GenSTAC. The first coaddition step (orange, left) reads in data and averages stellar position over $N$ frames for interpolation. Next, the actual align-and-stack step (green, right) outputs final images. Initiation and output (blue) are also included. "EOF" abbreviates "end-of-file". In this work, we choose $N$ during the GenSTAC initiation step, but this choice may be scripted for fully automated use.

stored as frames of $1024 \times 1024$ pixels in FITS datacubes with a pixel scale of 0 ." 043 .

\subsection{Generalized Tracking and Correction Pipeline}

We introduce a new pipeline, GenSTAC, to handle laseronly targets. The standard Robo-AO pipeline shifts-and-adds based upon cross-correlation to a diffraction-limited PSF. GenSTAC operates under the assumption of a large Gaussian PSF due to the lack of tip-tilt correction, and hence shifts-andadds each frame based on averaged stellar positions over many frames. This enables laser-only correction on faint targets and faster operation on bright targets. When the average stellar position over $N$ frames is reduced to $N=1$, the original pipeline outperforms GenSTAC because GenSTAC does not cross-correlate with a diffraction-limited PSF. When GenSTAC finds the best solution to be $N=1$ as described below (i.e., for $m_{V}<15.5$ targets), it reverts to the original pipeline for full tiptilt correction.

GenSTAC shifts-and-adds frames according to the following steps, summarized in Figure 2.

1. Raw frames are read into GenSTAC.

2. Choose the number of frames $N$ to bin together for averaged stellar positions. When scripted, multiple values of $N$ are tested and the best resulting FWHM determines the best $^{5} N$. Otherwise, the user chooses $N$ directly.

GenSTAC binning is usually performed based upon the brightness of the target star. Extremely faint objects, producing less than one photon per frame, are binned on

\footnotetext{
5 Using FWHM to determine the best $N$ rather than magnitude avoids any danger from incorrect catalog magnitudes.
}

second-or-longer timescales to remove long-term tracking drifts. Brighter objects enable faster operation with improved performance using some tip/tilt information.

To quantify the effective seeing of laser-only AO for 15000 targets, however, $N$ is specified by the user in order to bin away all tip-tilt information regardless of target brightness.

3. A first-pass through the frames is performed. The average position of the star for each group of $N$ frames throughout the observation is recorded during this first coaddition step.

4. The averaged pixel positions are interpolated with onedimensional cubic splines.

5. The frames are dark-subtracted, flat-fielded, shifted, and added to produce a final image. Shifting uses the output of the cubic interpolation in the previous step. While the standard Robo-AO bright-star pipeline (Law et al. 2014) employs the Drizzle algorithm (Fruchter \& Hook 2002), Drizzle is not applied by GenSTAC due to the assumption of large Gaussian PSFs.

\subsection{Measuring Performance}

Once all frames are reduced into a final image, we use three measures to characterize the quality of each observation: the full-width at half-maximum (FWHM) diameter, the $50 \%$ encircled energy $\left(\theta_{50}\right)$ diameter, and the Strehl ratio. FWHM is calculated as the diameter of the circle with an area of all pixels in the photometric aperture brighter than half the peak. $\theta_{50}$ is calculated as the diameter of the area containing half of the cumulative flux from the PSF. The Strehl is calculated as the total-flux-normalized peak intensity of the PSF divided by a theoretical diffraction-limited, normalized PSF. For the faint targets of the current paper, we are in the very low-Strehl regime, making the FWHM and $\theta_{50}$ more relevant in quantifying faint $\mathrm{AO}$ performance.

We break up 15000 separately targeted Robo-AO observations into 42000 images, typically of $30 \mathrm{~s}$ each. We desire to test laser-only image quality over a wide range of conditions; as the seeing evolves rapidly, these $30 \mathrm{~s}$ exposures represent independent realizations of the seeing. We then characterize the FWHM-, $\theta_{50^{-}}$, and Strehl- improvements in effective seeing. To ensure breaking up the 15000 observations in this way does not bias the seeing statistics, we compared median FWHM and $\theta_{50}$ using only one $30 \mathrm{~s}$ image per separately targeted observation and observed no change in seeing statistics from using 42000 realizations.

\section{Results}

We present measurements over the 15000-observation Robo-AO data set of 42000 independent realizations of the seeing, to show effective seeing for telescope-magnitudelimited targets. Although almost all Robo-AO observed targets were bright enough for tip-tilt correction (by design), we exclude all tip-tilt information from bright targets by binning frame-by-frame changes in position away in order to test laseronly AO performance on 15000 targets. We also run statistical samples of the data set over a range of binning timescales to quantify effective seeing as tip-tilt error is added, from guidestar correction all the way to laser-only correction. 
Table 1

Laser-only AO Results Summary

\begin{tabular}{|c|c|c|c|c|}
\hline & Laser-only (arcsec) & Seeing-limited $(\operatorname{arcsec})$ & Improvement $(\%)$ & Laser/Seeing Ratio \\
\hline FWHM & $0.6 \pm 0.3$ & $1.1 \pm 0.5$ & $39 \% \pm 19 \%$ & $0.6 \pm 0.2$ \\
\hline$\theta_{50}(50 \%$ enc. $)$ & $1.1 \pm 0.5$ & $1.5 \pm 0.5$ & $23 \% \pm 16 \%$ & $0.8 \pm 0.2$ \\
\hline Strehl & $0.024 \pm 0.017$ & $0.010 \pm 0.007$ & $\ldots$ & $2.1 \pm 1.1$ \\
\hline
\end{tabular}

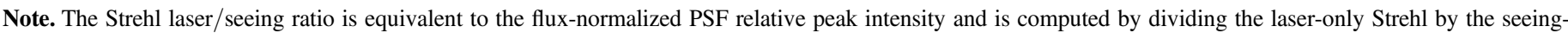

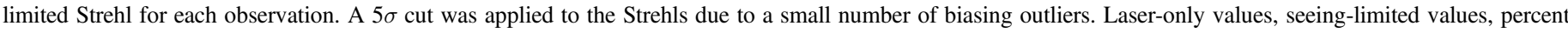

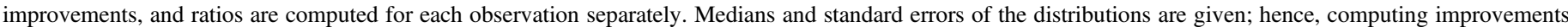
directly from the quoted measurements will produce values different from the ones above.

\subsection{System Performance and Resolution Improvement with Laser-only Correction}

For a $1.2 \mathrm{~s}$ binning timescale, simulating an autoguiderequipped Robo-AO for observing a faint star, we compute the seeing-limited and laser-only FWHM, $\theta_{50}$, and Strehl of 14954 separately targeted observations, with $4275230 \mathrm{~s}$ images processed by GenSTAC. Seeing-limited performance is measured from the $20 \mathrm{~s}$ acquisition image for each observation. Observations with technical problems that would otherwise bias our data were removed from this list with $5 \sigma$-clips, leaving 14158 targets and 40521 images. The resulting distributions (summarized in Table 1), described by median value and standard deviation, are as follows.

Seeing-limited FWHM is 1 !" $1 \pm 0$ ". 5 , while laser-only AO FWHM is $0 . ! 6 \pm 0 . ! 3$, an improvement of $39 \% \pm 19 \%$. Seeing-limited $\theta_{50}$ is $1 . " 5 \pm 0$ ". 5 , while laser-only AO $\theta_{50}$ is 1 !" $1 \pm 0 . " 5$, an improvement of $23 \% \pm 16 \%$. Strehls also showed improvement, although Strehl contrast in the fainttarget regime becomes less relevant due to lower values. Laseronly Strehl is $0.024 \pm 0.017$, while the seeing-limited Strehl is $0.010 \pm 0.007$. Strehl improvement over the seeing-limit and the associated uncertainty are large due to the non-linear peak effects of concentrating photons into a smaller area. All 42000 individual observations are plotted against each other in Figure 3. Scatterplot points are 2D-binned, interpolated, and displayed as AO-resolution versus seeing-resolution contours.

As a check against implicit tuning toward bright targets in the Robo-AO data set, we examine improvement-versusbrightness contour-scatterplots in Figure 4, which remains relatively constant across magnitude. For faint targets close to the background-noise level, measurement of resolution becomes slightly less accurate, with a small apparent increase in improvement, although not at a significant-enough level to obscure the constant-improvement trend.

\subsection{Sensitivity of Improvement to Guiding Timescale}

In addition to laser-only correction, we measure AO correction with varying amounts of tip-tilt present. Stacking more frames progressively removes tip-tilt information. In Figure 5, we show that the FWHM performance drops to a steady value around one-second timescales, while the $\theta_{50}$ remains approximately constant across timescales.

We randomly selected several observations of $\theta_{50}$ median improvement and measured normalized cumulative flux as a function of radius away from PSF center. For each target, the cumulative flux distribution for full-AO, partial tip-tilt "hybrid"-AO, and seeing-limited tracking were compared. A $0.6 \mathrm{~s}$ guiding timescale was chosen as representative of "hybrid" tracking. While seeing-limited cumulative flux always

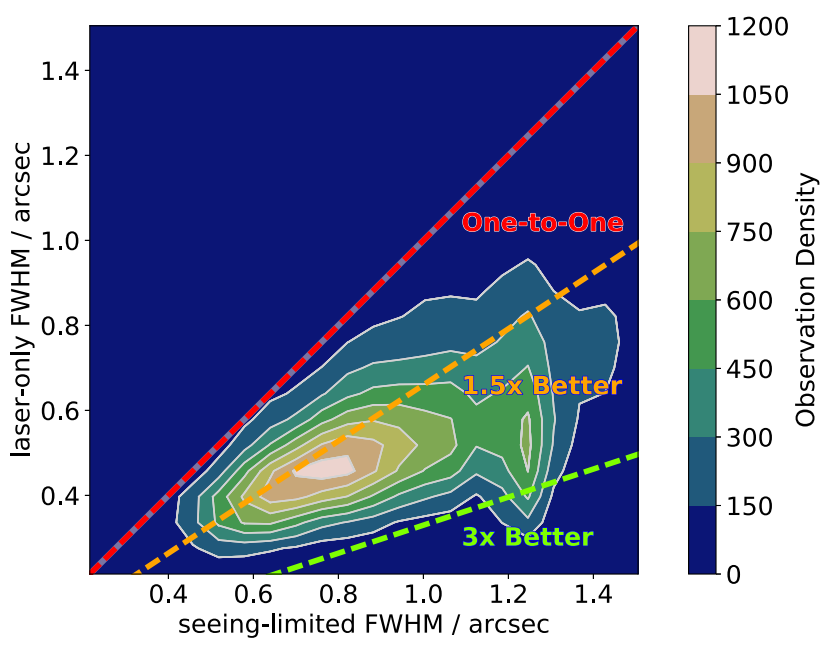

(a) FWHM Comparison

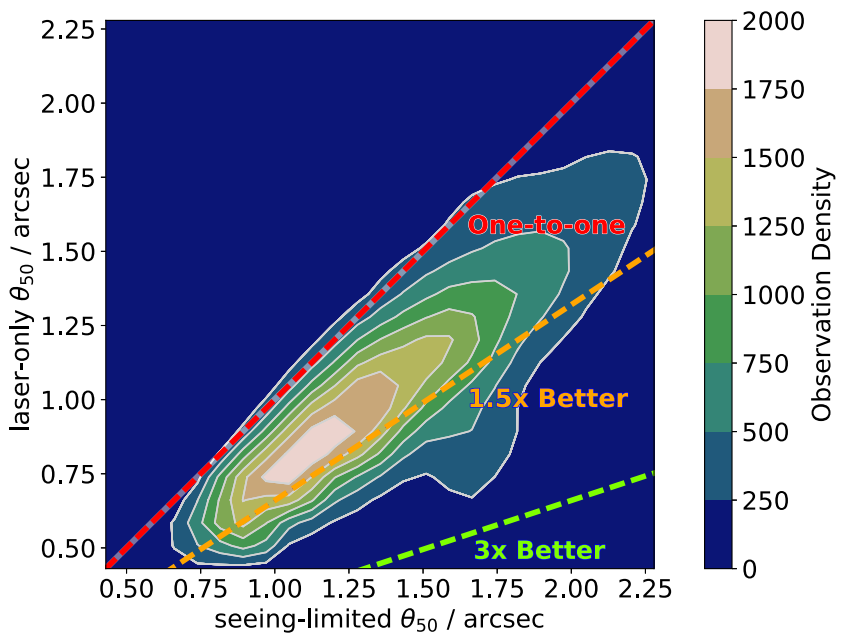

(b) $\theta_{50}$ Comparison

Figure 3. Performance contour plots of laser-only AO $(Y / \operatorname{arcsec})$ vs. seeinglimited performance ( $X / \operatorname{arcsec}) .42000$ raw scatterplot points were 2D-binned, interpolated, and contour-plotted. Reference lines of increasing FWHM $_{\text {seeing }}$ / FWHM $_{\text {laser-only }}$ are drawn to guide the eye. These include no increase (red) $1.5 \times$ (orange), and $3 \times$ (magenta).

converges last, both full-AO and "hybrid"-AO distributions quickly converge and have nearly identical $\theta_{50}$, within $\sim 0$ ". 1 of each other. Within the $50 \%$ encircled energy radius, the contributions from the PSF core to the cumulative distribution, which occur at very small radii, are dominated by the roughly linear increase in PSF flux that scales as enclosed-area. This agrees with the median $\theta_{50}$ obtained from each larger guidingtimescale sample: a diffraction-limited $0.1 \mathrm{~s}$ timescale median $\theta_{50}$ of 1 ". 04 is only 0 " 1 better than a $1.2 \mathrm{~s}$ autoguider timescale 


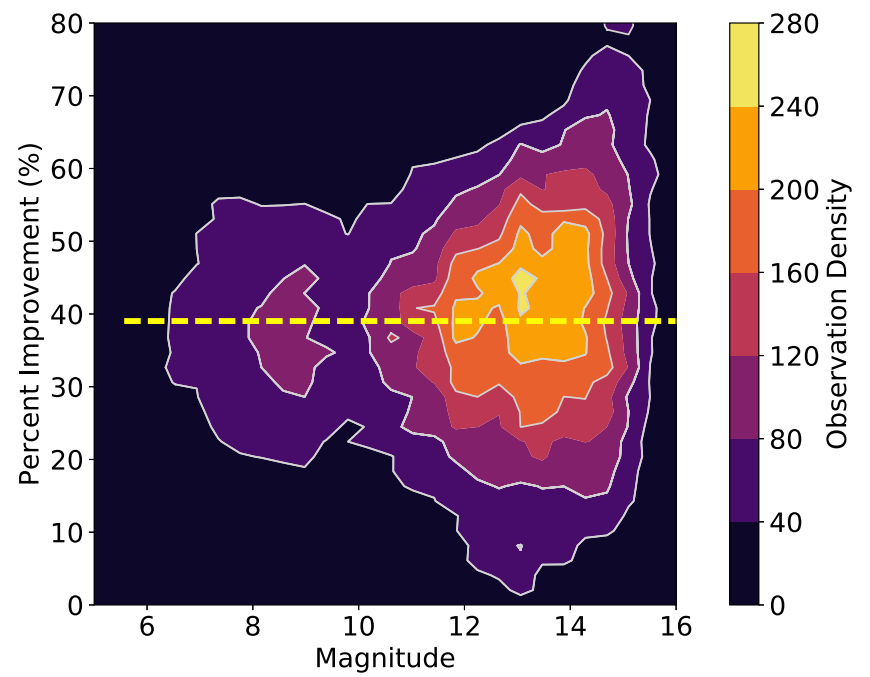

(a) FWHM Improvement versus Magnitude

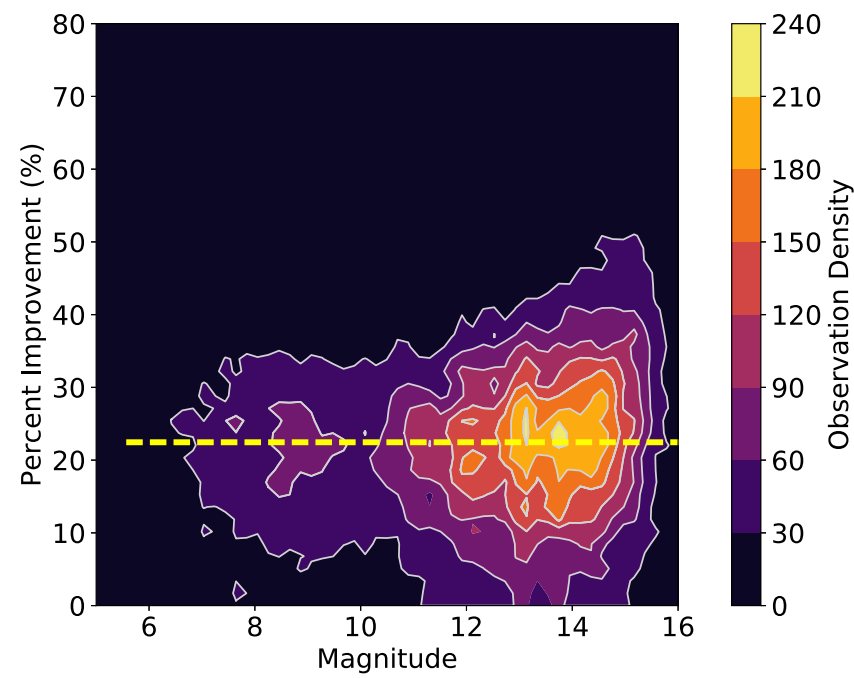

(b) $\theta_{50}$ Improvement versus Magnitude

Figure 4. Laser-only improvement vs. magnitude. Though median improvement (yellow) remains constant across magnitude, the spread of the data increases toward the faint end due to the effects of noise while measuring resolution.

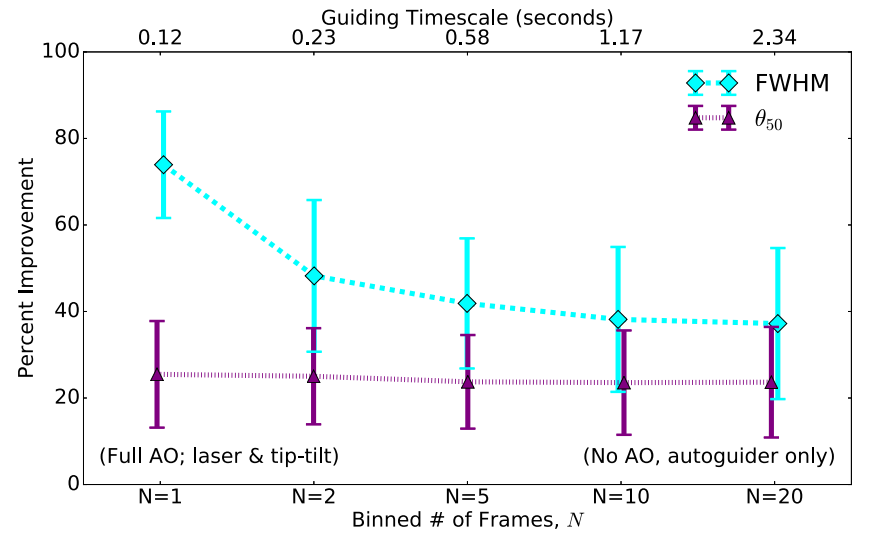

Figure 5. Improvement in effective seeing across guiding timescale. GenSTAC's guide-star mode lies to the left, where full tip-tilt information is employed, while the autoguider mode to the right uses no tip-tilt information. $3 \sigma$-clips are applied to the means of each FWHM distribution, as FWHM standard performance in "hybrid mode" is affected by failed measurements in the distribution wings. $\theta_{50}$ is more robust, requiring no cuts. median $\theta_{50}$ of 1 !' 15 . For reference, the median $\theta_{50}$ for slowguided, seeing-limited observations is $1 . " 5$.

The takeaway from this $50 \%$ encircled energy behavior is that for science goals requiring high-resolution encircled energy observations, a Robo-AO LGS system equipped with an autoguider and running the laser system should give comparable performance to full-AO correction. For faint targets especially, this greatly reduces the sky-coverage tip-tilt problem.

We also estimate the limiting magnitude for which each timescale is valid. Diffraction-limited performance with tip-tilt correction is possible to $\sim 15.5 \mathrm{mag}$. We estimate that GenSTAC provides some improvement by using residual tiptilt information down to $\sim 18 \mathrm{mag}$, and operates as a software slow-guider for targets down to the limiting magnitude of the instrument at a given exposure time.

Samples were randomly selected from 42000 observations and processed at characteristic timescales $(N)$.

Assuming approximately normal distributions, mean improvements for each timescale were obtained at a $99 \%$ confidence interval and margins-of-error of no more than $\sim 1.1$ pixel and $\sim 2 \%$ for resolutions (FWHM and $\theta_{50}$ ) and percent improvements, respectively. A $3 \sigma$ cut to FWHM was applied to each sample, removing up to $10 \%$ of observations. Without this cut, mean FWHM improvement is affected by occasional observations with technical problems, where telescope shake was present, or the PSF of a binary was measured assuming a non-binary. The margin-of-error for FWHM and $\theta_{50}$ remained less than $\sim 1.1$ pixel without any $\sigma$-clipping. Figure 6 verifies our assumption of normal or skewed-normal distributions for each measurement, such that $\sigma$-clipping does not remove standard system performance.

\section{Discussion and Conclusions}

For our LGS AO system, Robo-AO, the effective seeing upon arbitrarily faint targets demonstrates $39 \% \pm 19 \%$ improvement above the seeing-limit, even in the absence of tip-tilt correction. Faint targets with some recoverable tip-tilt information show corresponding gains in seeing, culminating in diffraction-limited performance for moderately bright guidestar targets. Our FWHM results on an intermediate-class telescope show a $2 \times$ to $3 \times$ effective-seeing improvement similar to those of the LGS $+\mathrm{P} 1$ mode upon the Gemini North telescope. Due to the lack of tip-tilt correction, laser-only AO does not require large apertures. Furthermore, whereas LGS $+\mathrm{P} 1$ improvements have been demonstrated in the NIR for $10^{2}$ targets (Trujillo et al. 2013), our LGS without tip-tilt improvement holds for $10^{4}$ targets, into the visible.

$50 \%$ encircled energy demonstrates for $10^{4}$ targets the same improvement upon both diffraction-limited tip-tilt correction and slow-guiding correction where tip-tilt correction is no longer possible, in agreement with Davies et al. (2008). These results suggest that intermediate-class telescopes equipped with LGS AO systems will see significant gains in performance upon faint targets when running the laser with a slow-guiding drift corrector, especially upon targets for which we most care about the encircled energy. For example, the proposed Rapid Transient Surveyor instrument (RTS) combines an integral field spectrograph (IFS) placed behind an LGS AO system on the robotized University of Hawai'i $2.2 \mathrm{~m}$ telescope. RTS will enable precise mapping of dark matter in the local universe by characterizing SN1a in the IR, which requires IFS for faint 

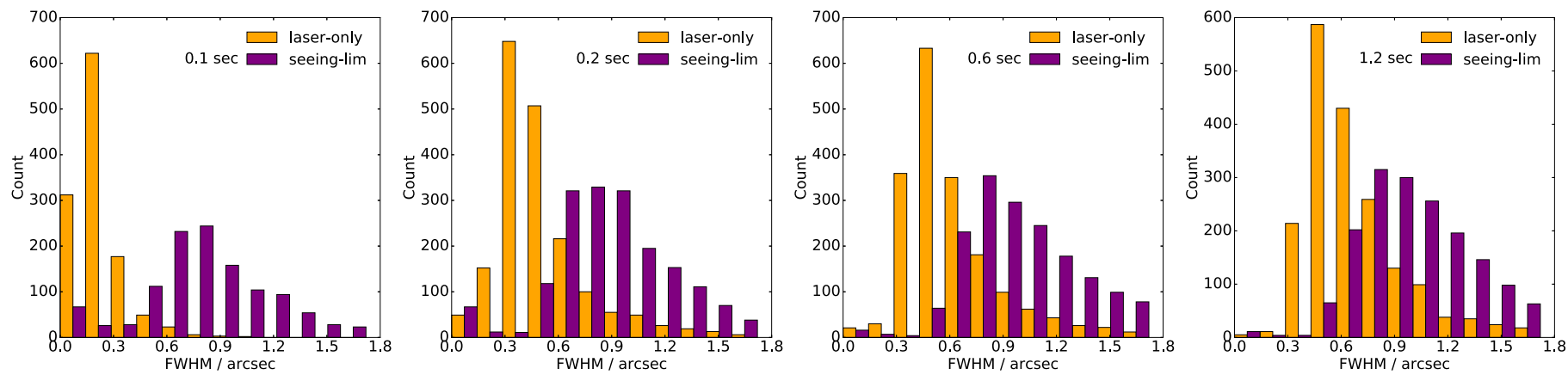

(a) Laser-off / Laser-on FWHM Distributions
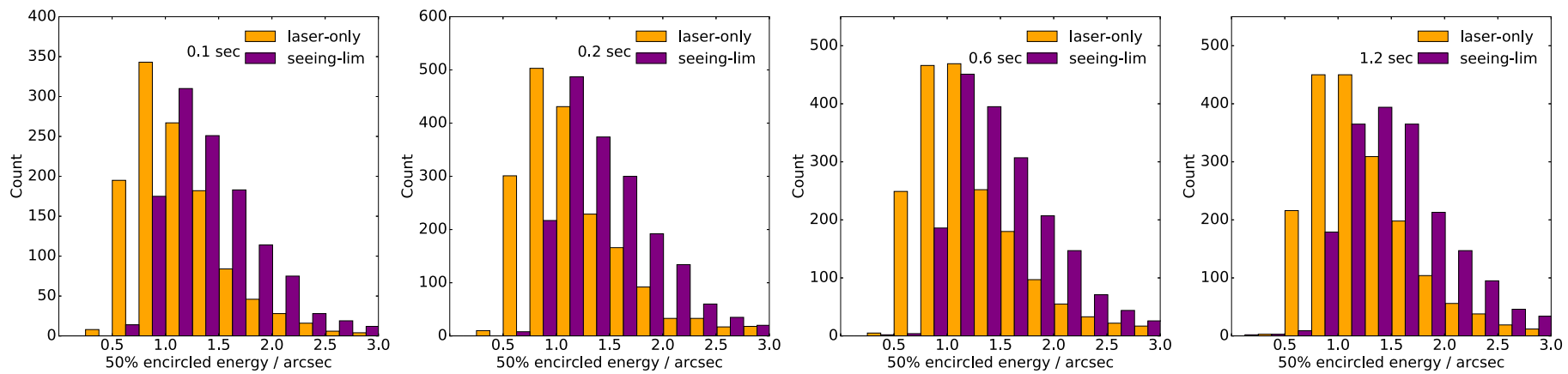

(b) Laser-off / Laser-on $\theta_{50}$ Distributions
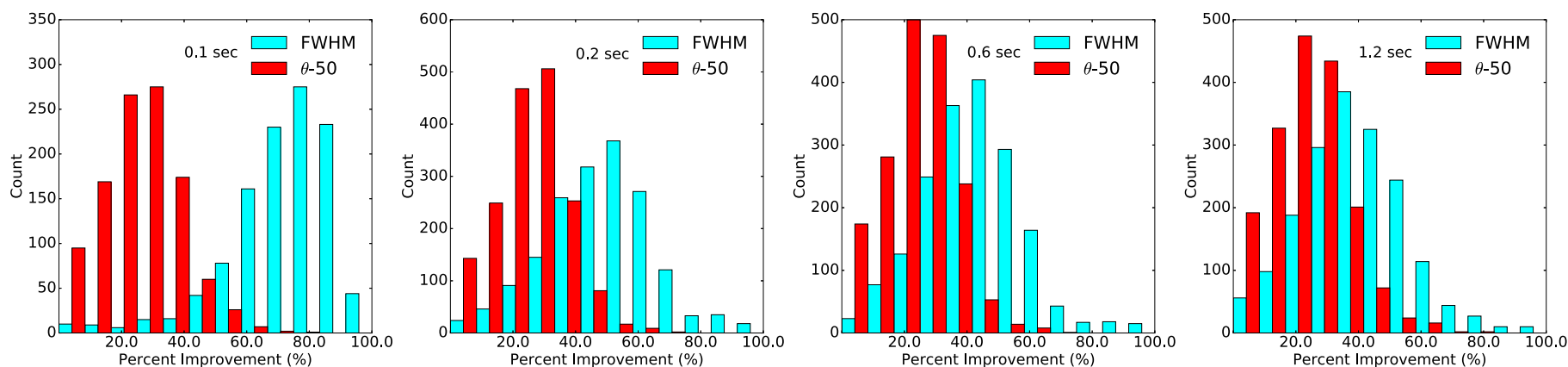

(c) FWHM and $\theta_{50}$ Improvement Distributions

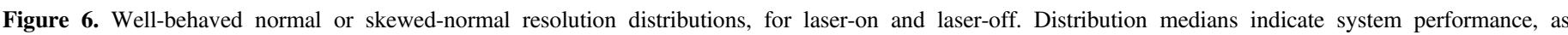

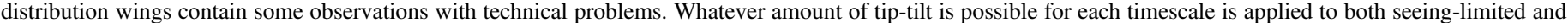
AO-images, resulting in slightly underestimated improvement for $0.1-0.6 \mathrm{~s}$ timescales.

sources (Baranec et al. 2016a). Our $\theta_{50}$ results motivate future exploration of laser-only IFS for those RTS targets without sufficiently bright guide stars.

We investigate laser-only AO with GenSTAC, a new RoboAO pipeline. GenSTAC provides post-processing options for guide-star correction, partial tip-tilt correction, and laser-only correction. GenSTAC binning on timescales longer than $1 \mathrm{~s}$ serves as a software equivalent to a slow-guider mounted to the telescope by converting stars that are too faint for guide-star correction into adequate stars through averaging and interpolating procedures described in Section 2.2. For moderately faint targets, it extracts any remaining tip-tilt information; very faint targets with no tip-tilt information will rely purely on laser correction.

In future work, we employ laser-only correction to search hundreds of faint KOIs $\left(m_{\text {Kepler }}>15.7\right)$ for stellar blends with Robo-AO and compare the companion fraction between the faint and bright KOI populations, especially for M-dwarfs.

As LGS AO systems continue to proliferate (Davies \& Kasper 2012), small-to-intermediate-class observatories with
LGS will benefit greatly from running the laser and a slowguider whenever observing faint targets beyond diffractionlimited sky-coverage.

We thank Rebecca Jensen-Clem for helpful discussions on the project.

This research has made use of the NASA Exoplanet Archive, which is operated by the California Institute of Technology, under contract with the National Aeronautics and Space Administration under the Exoplanet Exploration Program.

This research made use of Astropy, a community-developed core Python package for Astronomy (Astropy Collaboration et al. 2013), and the NumPy, SciPy, and Matplotlib Python modules (Jones et al. 2001; Oliphant 2007; Millman et al. 2011; Hunter 2007; van der Walt et al. 2011).

This work was partially supported by NASA XRP Grant NNX15AC91G. The Robo-AO system is supported by collaborating partner institutions, the California Institute of Technology and the Inter-University Centre for Astronomy and Astrophysics, and by the National Science Foundation under 
grant Nos. AST-0906060, AST-0960343, and AST-1207891, by the Mount Cuba Astronomical Foundation, and by a gift from Samuel Oschin. C.B. acknowledges support from the Alfred P. Sloan Foundation. We are grateful to the Palomar Observatory staff for their support of Robo-AO on the $1.5 \mathrm{~m}$ telescope, particularly S Kunsman, M Doyle, J Henning, R Walters, G Van Idsinga, B Baker, K Dunscombe, and D Roderick.

This research was carried out on the traditional lands of the Occaneechi, Haw, and Eno Native American tribes in Orange County, NC, and on the traditional lands of the Luiseño, or Payómkawichum, at Palomar Observatory, CA.

Finally, we would like to thank the anonymous referee who graciously gave their time to make this the best version of this work.

\section{ORCID iDs}

Ward S. Howard (iD https://orcid.org/0000-0002-0583-0949

Nicholas M. Law (i) https://orcid.org/0000-0001-9380-6457

Carl A. Ziegler (iD https://orcid.org/0000-0002-0619-7639

Christoph Baranec (iD https://orcid.org/0000-0002-1917-9157

Reed Riddle (iD https://orcid.org/0000-0002-0387-370X

\section{References}

Astropy Collaboration, Robitaille, T. P., Tollerud, E. J., et al. 2013, A\&A, 558, A33

Baranec, C., Lu, J. R., Wright, S. A., et al. 2016a, Proc. SPIE, 9909, 99090F

Baranec, C., Riddle, R., Law, N. M., et al. 2014, ApJL, 790, L8
Baranec, C., Ziegler, C., Law, N. M., et al. 2016b, AJ, 152, 18

Chabrier, G. 2003, PASP, 115, 763

Davies, R., \& Kasper, M. 2012, ARA\&A, 50, 305

Davies, R., Rabien, S., Lidman, C., et al. 2008, Msngr, 131, 7

Dressing, C. D., \& Charbonneau, D. 2015, ApJ, 807, 45

Foy, R., \& Labeyrie, A. 1985, A\&A, 152, L29

Fruchter, A. S., \& Hook, R. N. 2002, PASP, 114, 144

Gratadour, D., Mugnier, L. M., \& Rouan, D. 2005, A\&A, 443, 357

Guillaume, M., Melon, P., Refregier, P., \& Llebaria, A. 1998, JOSAA, 15,2841

Haas, M. R., Batalha, N. M., Bryson, S. T., et al. 2010, ApJL, 713, L115

Hardy, J. W. 1998, Adaptive Optics for Astronomical Telescopes (New York: Oxford Univ. Press), 448

Hunter, J. D. 2007, CSE, 9, 90

Jensen-Clem, R., Duev, D. A., Riddle, R., et al. 2018, AJ, 155,

Johnson, J. A., Apps, K., Gazak, J. Z., et al. 2011, ApJ, 730, 79

Jones, E., Oliphant, T., Peterson, P., et al. 2001, SciPy: Open Source Scientific Tools for Python, http://www.scipy.org/

Koch, D. G., Borucki, W. J., Basri, G., et al. 2010, ApJL, 713, L79

Law, N. M., Morton, T., Baranec, C., et al. 2014, ApJ, 791, 35

Millman, K. J., \& Aivazis, M. 2011, CSE, 13, 9

O’Donovan, F. T., Charbonneau, D., Torres, G., et al. 2006, ApJ, 644, 1237

Oliphant, T. E. 2007, CSE, 9, 10

Rigaut, F., \& Gendron, E. 1992, A\&A, 261, 677

Roberts, S., \& Singh, G. 1998, Proc. SPIE, 3353, 611

Snyder, D. L., \& Schulz, T. J. 1990, JOSAA, 7, 1251

Steinbring, E., Faber, S. M., Macintosh, B. A., Gavel, D., \& Gates, E. L. 2005 , PASP, 117,847

Trujillo, C., Ball, J., Boccas, M., et al. 2013, in Proc. 3rd AO4ELT Conf., ed. S. Esposito \& L. Fini (Firenze, Italy: INAF-Observatorio Astrofisico de Arcetri), 51

van der Walt, S., Colbert, S. C., \& Varoquaux, G. 2011, CSE, 13, 22

Wizinowich, P., Smith, R., Biasi, R., et al. 2014, Proc. SPIE, 9148, 91482B

Ziegler, C., Law, N. M., Morton, T., et al. 2017, AJ, 153, 66 